Mathematisches Forschungsinstitut Oberwolfach

Report No. 35/2020

DOI: $10.4171 /$ OWR/2020/35

\title{
Mini-Workshop: Dimers, Ising and Spanning Trees beyond the Critical Isoradial Case
}

\author{
Organized by \\ Cédric Boutillier, Paris \\ Sanjay Ramassamy, Gif-sur-Yvette \\ Marianna Russkikh, Cambridge MA
}

15 November - 21 November 2020

\begin{abstract}
The goal of this mini-workshop is to gather specialists of the dimer, Ising and spanning tree models around recent and ongoing progress in two directions. One is understanding the connection to the spectral curve of these models in the cases when the curve has positive genus. The other is the introduction of universal embeddings associated to these models. We aim to use these new tools to progress in the study of scaling limits.
\end{abstract}

Mathematics Subject Classification (2010): 82B20, 14H70, 52C26, 30G25, 53A70, 13F60, 51A20.

\section{Introduction by the Organizers}

The dimer model is a probabilistic model of random perfect matchings on graphs. In the case of planar graphs, various mappings lead to consider the Ising model for magnetization and the spanning tree model as special cases of the dimer model. While these models have been studied for almost a century, there has been a flurry of recent research activity on these topics warranting the need of a meeting to assess the state-of-the-art and discuss the perspectives.

More specifically, the goal of this mini-workshop was to gather specialists of the dimer, Ising and spanning tree models around recent and ongoing progress in two main directions. One is the introduction of universal embeddings associated to these models. The other is understanding the connection to the spectral curve of these models in the cases when the curve has positive genus. Due to the Covid-19 pandemic the meeting had to be held entirely online. Still, with one mini-course, ten lectures and two discussion sessions we managed to foster sustained interactions between the participants. 
Dmitry Chelkak provided a three-hour mini-course on the recently introduced t-embeddings, p-embeddings and s-embeddings, which provide the right setup for the definition of discrete holomorphic functions on arbitrary periodic bipartite graphs and for the convergence to conformally invariant objects in the scaling limit for the bipartite dimer model and the Ising model. Some of the theory, such as the existence of p-embeddings and of discrete three-dimensional objects related to them, is still an area of ongoing research. The application of such embeddings to random planar maps is a major long-term goal.

In a similar spirit, Asaf Nachmias showed the convergence of discrete holomorphic functions on orthodiagonal embeddings of arbitrary graphs to their continuous counterparts. Paul Melotti described a local move on alpha-embeddings, which are a common generalization of s-embeddings and orthodiagonal embeddings. Niklas Affolter presented local moves for several geometric configurations based on cluster algebra mutations, recovering as special cases s-, t- and orthodiagonal embeddings.

Cluster algebras also appeared in the talk of Terrence George, where their action on networks induces an integrable system linearized on the Prym variety of the spectral curve. Vladimir Fock presented a universal construction for the tau function on Riemann surfaces, with an application to the dimer cluster integrable system. David Cimasoni described properties of a Kasteleyn operator in terms of elliptic functions on minimal graphs and its application to the inverse spectral problem for planar periodic bipartite dimers for genus 1 Harnack curves, and the construction of Gibbs measures beyond the periodic case.

Beyond the two special topics of embeddings and spectral maps, new developments were reported on planar statistical mechanics models. Marcin Lis showed a computation of boundary and bulk correlations for the Ashkin-Teller model, a generalization of the Ising model, via the introduction of a random current representation. Nathanaël Berestycki described a dimer model on the upper half-plane with free boundary conditions, which gives rise to a Gaussian free field with free or Neumann boundary conditions. Richard Kenyon introduced the multidimer model with first results on the free energy and fluctuations of the edge process. Finally Zhongyang Li proved two conjectures of Benjamini and Schramm for site percolation in the case of transitive hyperbolic graphs.

The organizers are grateful to the MFO for help in organizing the mini-workshop, to Paul Melotti for acting as the video conference assistant and to Niklas Affolter for putting together the present report. We also thank the ANR DIMERS project (grant number ANR-18-CE40-0033) for accepting to fund transportation costs if the workshop had been held at the MFO.

Acknowledgement: The MFO and the workshop organizers would like to thank the National Science Foundation for supporting the participation of junior researchers in the workshop by the grant DMS-1641185, "US Junior Oberwolfach Fellows". Moreover, the MFO and the workshop organizers would like to thank the Simons Foundation for supporting NAME(S) in the "Simons Visiting Professors" program at the MFO. 
Mini-Workshop: Dimers, Ising and Spanning Trees beyond the Critical Isoradial

Case

\section{Workshop: Mini-Workshop: Dimers, Ising and Spanning Trees beyond the Critical Isoradial Case}

\section{Table of Contents}

Nathanaël Berestycki (joint with Marcin Lis and Wei Qian)

Dimers with free boundary: random walk representation and scaling limit

Marcin Lis

On boundary correlations in planar Ashkin-Teller models ........... 8

Zhongyang Li

Percolation in the Hyperbolic Plane and Benjamini-Schramm Conjecture

Dmitry Chelkak

$S$-/t-/p-embeddings: state-of-the-art of convergence results for the bipartite dimer and Ising models on irregular planar graphs .........

Vladimir Fock

Tau-function on Riemann surfaces.

David Cimasoni (joint with Cédric Boutillier and Béatrice de Tilière)

Elliptic dimer models and genus 1 Harnack curves

Richard Kenyon (joint with Andrei Pohoata)

The multidimer model

Niklas Affolter (joint with Max Glick, Pavlo Pylyavskyy and Sanjay Ramassamy)

Triple crossing diagrams, projective configurations, dimers

Asaf Nachmias (joint with Ori Gurel-Gurevich and Daniel C. Jerison)

The Dirichlet problem for orthodiagonal maps

Paul Melotti (joint with Sanjay Ramassamy and Paul Thévenin)

Cube flips in s-embeddings and $\alpha$-immersions

Terrence George

Inverse spectral problem for biperiodic networks 

Mini-Workshop: Dimers, Ising and Spanning Trees beyond the Critical Isoradial

Case

Abstracts

\title{
Dimers with free boundary: random walk representation and scaling
} limit

\author{
NATHANAËL BERESTYCKI \\ (joint work with Marcin Lis and Wei Qian)
}

We study the dimer model in which particles on part of the boundary are allowed to form monomers with some fixed weight $z>0$ called the monomer fugacity. A bijection described in Giuliani, Jauslin and Lieb [1] relates this model to a non-bipartite dimer, whose corresponding Kasteleyn matrix describes a random walk with negative rates along the boundary. Yet under certain assumptions on the domain boundary, we prove an effective random walk representation for the inverse Kasteleyn matrix. The proof makes use of the Schur complement formula and the exact computation of the potential kernel of an auxiliary one-dimensional walk. The assumptions are in particular satisfied in the infinite volume (thermodynamic) limit on the upper half plane. In this case, we further show that the scaling limit of the height function is given by a Gaussian free field with free (or Neumann) boundary conditions, thereby answering a question of Giuliani et al. To our knowledge, this is the first occurrence of this field as a scaling limit in the study of height functions.

Free boundary dimers (aka monomer-dimer model). Let $G=(V, E)$ be a finite planar bipartite graph which is a subgraph of $\mathbb{Z}^{2}$. Let $\partial_{m}$ be a chosen subset of boundary vertices (i.e., the vertices adjacent to the unique unbounded external face). A monomer-dimer cover of $G$ is a set $M \subset E \cup \partial_{m}$ such that for each vertex $v \in V$, either $v \in M$, or $v$ belongs to exactly one edge in $M$. Let $\mathcal{M D}(G)$ be the set of all MD covers of $G$, and let $\mathcal{D}(G)$ be the set of all dimer covers, i.e. MD covers $M$ such that $M \cap \partial_{m}=\emptyset$. For $M \in \mathcal{M D}(G)$ we call $M \cap \partial_{m}$ to be the set of monomers of the configuration $M$. The monomer-dimer model is a random choice of a $\mathrm{MD}$ cover from $\mathcal{M D}(G)$ according to the following probability measure:

$$
\mathbf{P}(M)=\frac{z^{\# \text { monomers }}}{Z} .
$$

where \#monomers is of course the same as $\left|M \cap \partial_{m}\right|$, and $Z$ is a normalizing constant. We will always assume for convenience (in reality, this is not strictly necessary) that the graph is dimerable meaning $\mathcal{D}(G) \neq \emptyset$. In particular, the partition function satisfies $Z>0$. The height function of a monomer-dimer cover is a function defined (up to a constant) on the bounded faces of $G$. Its definition is identical to the dimer model. The main question we aim to address in this work is the scaling limit of the height function.

Boundary conditions. First, we assume that $G$ is a subset of the square lattice $\mathbb{Z}^{2}$; we assume without loss of generality that $0 \in V$ and is a black vertex. (This fixes a unique black/white partition of $G$.) We also assume that its vertex set $V$ is contained the upper half plane $\mathbb{H}=\{z \in \mathbb{C}: \Im(z) \geq 0\}$. Furthermore, $\partial_{m}=V \cap \mathbb{R}$, 
so the monomers are allowed exactly on the real line. The leftmost and rightmost vertices of $V \cap \mathbb{R}=\partial_{m}$ will be referred to as the corners of $G$. Finally, we assume that $G$ has at least one black corner and one white corner.

Main results. The monomer-dimer model as discussed above was discussed (with minor modifications) in a paper of Giuliani, Jauslin and Lieb. In that paper it was shown that the partition function $Z$ can be computed as a Pfaffian of a certain matrix $K$. Furthermore, a bijection was provided to a non-bipartite dimer model (the authors indicate that this bijection was suggested by an anonymous referee). A key result of our work is a random walk representation for the inverse Kasteleyn matrix associated to this model. Suppose $G$ is a graph satisfying the above assumption. Fix $z>0$ and assign weight $z$ to every monomer on $\partial_{m}$ except at either corner, where (for technical reasons) we choose the weight of a monomer to be given by $z^{\prime}=\frac{z}{2}+\sqrt{1+\frac{z^{2}}{4}}$. For $k \in \mathbf{N}=\{0,1, \ldots\}$ let us call $V_{k}=\{v \in V: \Im(v)=k\}$, so $\partial_{m}=V_{0}$. Let us call $V_{\text {even }}=V_{0} \cup V_{2} \cup \ldots$ and $V_{\text {odd }}=V_{1} \cup V_{3} \cup \ldots$

Theorem 1 (Random walk representation for inverse Kasteleyn matrix). There exists a pair of random walks $\left(Z_{\text {ev }}, Z_{\text {odd }}\right)$ on the state spaces $V_{\text {even }}$ and $V_{\text {odd }}$ respectively such that the following holds. Consider the monomer-dimer model on $G$ where the monomer weight $z>0$ on $V_{0}$ except at its corners where the monomer weight is $z^{\prime}$ as above. Let $K$ be the associated Kasteleyn matrix, and $\mathcal{L}=K^{*} K$, so that $K^{-1}=\mathcal{L}^{-1} K^{*}$. Then if $x, y \in V$,

$$
\mathcal{L}^{-1}(x, y)= \begin{cases}g_{\text {odd }}(x, y) & \text { if } x, y \in V_{\text {odd }} \\ (-1)^{\Re(y-x)} g_{\text {ev }}(x, y) & \text { if } x, y \in V_{\text {even }}\end{cases}
$$

where $g_{\text {ev }}, g_{\text {odd }}$ are the Green functions of $Z_{e v}$ and $Z_{\text {odd }}$ respectively, normalised by $\mathcal{L}(y, y)$.

Both $Z_{\mathrm{ev}}$ and $Z_{\text {odd }}$ behave like simple random walk far away (at distance more than 2) from the boundary vertices, but with jumps of size \pm 2 , so the parity of the walk does not change. Both have nontrivial boundary conditions, including some reflecting and absorbing boundary arcs along the non-monomer part of the boundary. Furthermore, both are allowed to make additional jumps along their bottommost vertices ( $V_{0}$ for $Z_{\mathrm{ev}}$ and $V_{1}$ for $Z_{\text {odd }}$ ). These jumps are symmetric, bounded in the even case but not the odd case, although they do have exponentially decaying tail. Hence in the scaling limit, these walks would converge to Brownian motion in the upper half plane $\mathbb{H}$ with reflection on the real axis and whatever boundary condition is inherited from the Neumann/Dirichlet status of the other boundary arcs. The above theorem allows us to describe in detail first an infinite volume (local) limit, then a scaling limit for the height function.

Proposition 2 (Infinite volume limit). Let $G_{n}$ be a sequence of graph such that $G_{n}$ satisfies the above boundary condition, and $G_{n} \uparrow \mathbb{Z}^{2} \cap \mathbb{H}$. Let $\mu_{n}$ denote the law of the monomer-dimer model on $G_{n}$ (with weight $z>0$ per monomer and $z^{\prime}$ at the corners, as in Theorem 1). Then $\mu_{n}$ converges weakly as $n \rightarrow \infty$ to a law $\mu$ which describes a.s. a random monomer-dimer configuration on $\mathbb{Z}^{2} \cap \mathbb{H}$. 
Mini-Workshop: Dimers, Ising and Spanning Trees beyond the Critical Isoradial Case
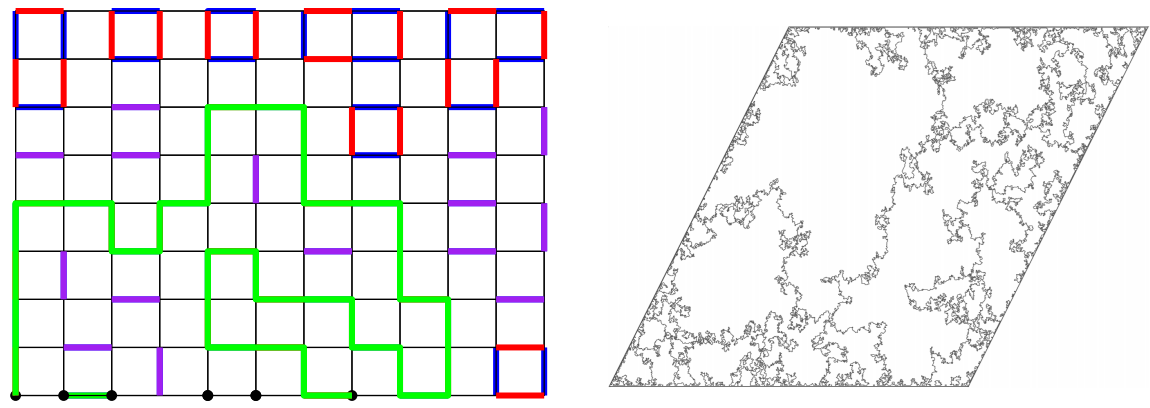

FiguRE 1. A superposition of two configurations, respectively blue and red. Double edges are in purple. The collection of arcs joining monomers to monomers is in green. Next, a simulation of $\mathrm{ALE}_{-\lambda, \lambda}$ by B. Werness.

To describe the scaling limit, let $\mathbb{H} g$ denote a Gaussian free field with Neumann (or free) boundary conditions. For a small $\epsilon>0$ (the mesh size), let $h^{\epsilon}$ denote the height function of the monomer-dimer model $\mu$ with weight $z$ in the infinite half plane $\mathbb{H} \cap \epsilon \mathbb{Z}^{2}$ (rescaled by $\epsilon$ ). We identify $h^{\epsilon}$ with an almost everywhere function $\mathbb{H}$ by taking the value of $h^{\epsilon}$ to be constant on each face, and view $h^{\epsilon}$ as a random distribution (modulo constant) on $\mathbb{H}$.

Theorem 3. Let $f_{1}, \ldots, f_{n} \in \mathcal{D}_{0}(\mathbb{H})$ be arbitrary test functions. Then as $\epsilon \rightarrow 0$, $\left(h^{\epsilon}, f_{i}\right)_{i=1}^{n} \rightarrow\left(\frac{1}{\sqrt{2} \pi} h_{\mathrm{GFF}}, f_{i}\right)_{i=1}^{n}$ in distribution.

A conjecture. In the study of the dimer model, a well known conjecture of Kenyon concerns the superposition of two independent dimer configurations. It is easy to check that such a superposition deterministically results in a gas of loops (including double edges) covering every vertex. Kenyon conjectured that this collection of loops converges in the scaling limit to $\mathrm{CLE}_{4}$, the conformal loop ensemble with parameter $\kappa=4$. This is strongly supported by the fact that in the continuum, $\mathrm{CLE}_{4}$ can be viewed as the level lines of a (Dirichlet) GFF with a specified variance. Major progress has been made recently on this conjecture through the work of Dubédat and Basok and Chelkak.

It is natural to ask if any interesting phenomenon occurs when we superpose two independent free boundary dimer configurations, say in the upper half-plane. For topological reasons, this gives rise to a gas of loops as above but also a collection of curves connecting monomers to monomers. See the figure above for an example. An obvious question is to describe the law of this collection $\mathcal{C}$ of curves in the scaling limit. By analogy with the above, and in view of our result (Theorem 3), it is natural to expect that this collection $\mathcal{C}$ converges in the scaling limit to the level lines of a GFF with Neumann boundary conditions on the upper-half plane. By a result of Qian and Werner, the law of these curves was determined to be the ALE $_{-\lambda, \lambda}$ process (ALE stands for Arc Loop Ensemble, even though they do not in fact contain loops). ALEs were defined by Aru, Sepulveda and Werner as 
certain local sets of the GFF (in fact, $\mathrm{ALE}_{-\lambda, \lambda}$ is characterised as the "thinnest" two-valued set of the GFF).

Conjecture 4. For any $z>0$, in the scaling limit, the collection of boundarytouching curves $\mathcal{C}$ converges to the Arc Loop Ensemble $A L E_{-\lambda, \lambda}$. Given the $A L E$, the loops form independent $C L E_{4}$ in each of the connected component of the complement.

\section{REFERENCES}

[1] Giuliani, Alessandro and Jauslin, Ian and Lieb, Elliott H., A Pfaffian Formula for Monomer-Dimer Partition Functions, Journal of Statistical Physics 163 (2016), 211-238.

\section{On boundary correlations in planar Ashkin-Teller models MARCIN LIS}

It has been well known since the work of Groeneveld, Boel and Kasteleyn [1] that in the Ising model the multi-point correlations of spins lying on the boundary of a planar graph are given by Pfaffians of the respective two-point correlations. This can be seen as the Wick's rule for expectation values of products of noninteracting Majorana fermions, and is one of the many manifestations of the fermionic structure underlying the planar Ising model. Recently it was noticed by the author that certain matrices of such boundary two-point functions are totally positive [2], i.e., determinants of all their minors are positive.

In this work we present a unified framework from which both the classical Pfaffian identities and total positivity inequalities of Ising boundary correlations are naturally concluded. In the proofs we use switching lemmas for random currents. The idea of using switching identities to establish Pfaffian relations of Ising correlations originated in the recent work of Aizenman, Duminil-Copin, Tassion and Warzel [3] which was an inspiration for our considerations.

More generally we derive linear identities and inequalities that are satisfied by boundary correlations of planar Ashkin-Teller models, i.e., two Ising-like spin configurations $\sigma$ and $\tilde{\sigma}$ coupled by a Hamiltonian with a four-body interaction. To this end we first define a random current representation of the model, and show a switching identity for the correlations of $\sigma$ and $\tilde{\sigma}$ which is a generalization of the classical switching lemma of Griffiths, Hurst, and Sherman [4] for two independent Ising models (the case of vanishing four body interactions). Subsequently we obtain a new switching identity for the correlations of the $\{-1,0,1\}$-valued spins $\varphi=(\sigma+\tilde{\sigma}) / 2$ and $\tilde{\varphi}=(\sigma-\tilde{\sigma}) / 2$. This yields a set of linear inequalities for the correlations of $\sigma$ and $\tilde{\sigma}$. Moreover, the desired linear identities follow from the crucial observation that the correlations of $\varphi$ and $\tilde{\varphi}$ may be forced to vanish by properly choosing the order of spin insertions on the boundary of a planar graph.

We also show that the same relations are satisfied by products of Pfaffians. Since the correlations of $\sigma$ and $\tilde{\sigma}$ factorize in the noninteracting case, a unified picture arises: The boundary correlations of $\sigma$ are given by Pfaffians of their respective two-point functions, whereas the mixed correlations of $\varphi$ and $\tilde{\varphi}$ are 
Mini-Workshop: Dimers, Ising and Spanning Trees beyond the Critical Isoradial Case

given by analogous determinants. The latter may be thought of as an instance of the fermionic Wick's rule for expectation values of products of noninteracting Dirac fermions.

This picture should be compared with the bosonic Wick's rule which states that higher moments of real Gaussian fields are hafnians and those of a complexified pair of independent Gaussian fields are permaments of their second moments. Interestingly, in our setting total positivity of two-point boundary correlations turns out to be intrinsically related to the first Griffiths inequality for the spins $\varphi$ and $\tilde{\varphi}$.

\section{REFERENCES}

[1] J. Groeneveld, R.J. Boel, P.W. Kasteleyn Correlation-function identities for general planar Ising systems, Physica A: Statistical Mechanics and its Applications 93 (1978), 138-154.

[2] M. Lis The Planar Ising Model and Total Positivity, Journal of Statistical Physics 166 (2017), 72-89.

[3] M. Aizenman, H. Duminil-Copin, V. Tassion, S. Warzel, Emergent planarity in twodimensional Ising models with finite-range Interactions, Inventiones Mathematicae 216 (2019), 661-743.

[4] R. B. Griffiths, C. A. Hurst, S. Sherman, Concavity of Magnetization of an Ising Ferromagnet in a Positive External Field, Journal of Mathematical Physics 11 (1970), 790-795.

[5] M. Lis On boundary correlations in planar Ashkin-Teller models, arXiv (2020), 2009.12131.

\section{Percolation in the Hyperbolic Plane and Benjamini-Schramm Conjecture \\ ZHONGYANG LI}

Introduced by Broadbent and Hammersley in 1957 (see [9]) to study the random spread of a fluid through a medium, percolation has been a celebrated model illustrating the phase transition.

Let $G=(V(G), E(G))$ be an infinite, locally finite, connected graph. A site percolation configuration $\omega \in\{0,1\}^{V(G)}$ is an assignment to each vertex in $G$ of either state 0 or state 1 . A cluster in $\omega$ is a maximal connected set of vertices in which each vertex has the same state in $\omega$. A cluster may be a 0-cluster or a 1-cluster depending on the common state of vertices in the cluster. A cluster may be finite or infinite depending on the total number of vertices in the cluster. We say that percolation occurs in $\omega$ if there exists an infinite 1-cluster in $\omega$. We can define bond percolation in a similar way by considering edge states in $\{0,1\}$ instead of vertex states.

A central question in percolation theory is when there exists an infinite cluster. The percolation model is a natural mathematical model for structure of matter, magnetization, or spread of pandemic diseases. See $[6,5]$ for overview on percolation.

The occurrence of percolation is closely related to the structure of the graph. A graph $G=(V(G), E(G))$ is called vertex-transitive, or transitive, if there exists a subgroup $\Gamma \subseteq \operatorname{Aut}(G)$, such that all the vertices are in the same orbit under the 
action of $\Gamma$ on $G$. The graph $G$ is called quasi-transitive if there exists a subgroup $\Gamma \subseteq \operatorname{Aut}(G)$, such that all the vertices are in finitely many different orbits under the action of $\Gamma$ on $G$.

The graph $G$ is called amenable if

$$
\inf _{K \subseteq V(G),|K|<\infty} \frac{\left|\partial_{E} K\right|}{|K|}=0,
$$

where $\partial_{E} K$ consists of all the edges in $E(G)$ that have exactly one endpoint in $K$ and one endpoint not in $K$. If the left-hand side of (1) is strictly positive, then the graph $G$ is called non-amenable.

A manifold $M$ is plane if every self-avoiding cycle splits it into two parts. We say the graph $G$ is planar if it can be drawn on the plane in such a way that its edges intersect only at their endpoints. We say that an embedded graph $G \subset M$ in $M$ is properly embedded if every compact subset of $M$ contains finitely many vertices of $G$ and intersects finitely many edges.

The number of ends of a connected graph is the supremum over its finite subgraphs of the number of infinite components that remain after removing the subgraph.

Of particular interest is the i.i.d. Bernoulli site (resp. bond) percolation on a graph. In such a model, an independent Bernoulli random variable, which takes value 1 with probability $p \in[0,1]$, is associated to each vertex (resp. edge). For the i.i.d. Bernoulli percolation, define

$$
\begin{aligned}
& p_{c}^{\text {site }}(G):=\inf \{p \in[0,1]: \operatorname{Bernoulli}(p) \text { site perc. on } G \text { has an infinite cluster a.s. }\} \\
& p_{u}^{\text {site }}(G):=\inf \{p \in[0,1]: \operatorname{Bernoulli}(p) \text { site perc. on } G \text { has a unique inf. cluster a.s. }\} \\
& p_{c}^{\text {bond }}(G):=\inf \{p \in[0,1]: \operatorname{Bernoulli}(p) \text { bond perc. on } G \text { has an infinite cluster a.s. }\} \\
& p_{u}^{\text {bond }}(G):=\inf \{p \in[0,1]: \operatorname{Bernoulli}(p) \text { bond perc. on } G \text { has a unique inf. cluster a.s. }\}
\end{aligned}
$$

Although percolation in the hypercubic lattice $\mathbb{Z}^{d}$, which is amenable, has been studied extensively, a lot of questions for percolation in more general graphs remain unknown. The research for percolation on general graphs was initiated in the paper [7] by Benjamini and Schramm, where they made several conjectures including the following two.

Conjecture 1. (Conjecture 7 of [7]) Suppose $G$ is planar, and the minimal degree in $G$ is at least 7 . Then at every $p$ in the range $\left(p_{c}^{\text {site }}(G), 1-p_{c}^{\text {site }}(G)\right)$, there are infinitely many infinite 1-clusters. Moreover, we conjecture that $p_{c}^{\text {site }}(G)<\frac{1}{2}$, so the above interval is nonempty.

Conjecture 2. (Conjecture 8 of [7]) Let $G$ be a planar graph. Let $p=\frac{1}{2}$ be the probability that a vertex is open and assume that a.s. percolation occurs in the site percolation on $G$, then almost surely there are infinitely many infinite 1-clusters.

Conjecture 1 was proved in [7] when the graph $G$ is obtained by adding to the binary tree edges connecting all pairs of nearest vertices of same level along a line. Conjecture 2 was proved in [7] when $G$ is a planar graph disjoint from the positive 
Mini-Workshop: Dimers, Ising and Spanning Trees beyond the Critical Isoradial Case

$x$-axis $(\{(x, 0): x>0\})$, such that every bounded set in the plane meets finitely many vertices and edges of $G$. Conjectures 1 and 2 were later proved in [8] when $G$ is a regular triangular tiling of the hyperbolic plane $\mathbb{H}^{2}$ in which each vertex has the same degree $d \geq 7$ and in [2] when $G$ is an infinite, connected, locally finite, transitive, planar graph by the following steps:

(1) By Proposition 2.1 of [3], an infinite, connected, locally finite, quasitransitive graph has either one or two or infinitely many ends. If the graph has two ends, then it is amenable. If it has infinitely many ends, then it is non-amenable.

(2) Prove that for i.i.d. Bernoulli percolation on any connected, locally finite, quasi-transitive graph with two ends, $p_{c}^{\text {site }}(G)=p_{u}^{\text {site }}(G)=1$.

(3) Prove that for i.i.d. Bernoulli percolation on any connected, locally finite, quasi-transitive graph with infinitely many ends, $p_{u}^{\text {site }}(G)=1$.

(4) By Theorem 2 of [4], for any planar graph $G$ with minimal vertex degree at least $7, p_{c}^{\text {site }}(G)<\frac{5+\sqrt{5}}{4(3+\sqrt{5})} \approx 0.3455$.

(5) By Lemma 3.5 of [1], the number of infinite clusters in any invariant percolation on any quasi-transitive, non-amenable planar graph with one end is a.s. 0,1 , or $\infty$. When $p \in\left(p_{c}, 1-p_{c}\right)$, to show that there exists infinitely many infinite 1-clusters, it suffices to exclude the case of a unique infinite 1-cluster.

(6) If the one-ended graph can be embedded into the plane such that the automorphism of the graph extended to isometries of the plane, the possibility of a unique infinite 1-cluster may be excluded by planar topology.

\section{REFERENCES}

[1] Benjamini, I. and Schramm, O. Percolation in the hyperbolic plane, Journal of the American Mathematical Society 14 (2000), 487-507.

[2] Li, Z. Site percolation on planar graphs, arXiv (2020), 2005.04529

[3] Babai, L. The growth rate of vertex-transitive planar graphs, Proceedings of the Eighth Annual ACM-SIAM Symposium on Discrete Algorithms, New Orleans (1997), 564-573.

[4] Halslegrave, J. and Panagiotis, C. Site percolation and isoperimetric inequalities for plane graphs, Random Structures and Algorithms 58 (2020), 150-163.

[5] Duminil-Copin, H. Sixty years of percolation, Proceedings of the ICM Rio (2018).

[6] Grimmett, G. Percolation, Springer (1999).

[7] Benjamini, I. and Schramm, O. Percolation beyond $\mathbb{Z}^{d}$, many questions and a few answers, Electronic Communications in Probability 1 (1996), 71-82.

[8] Li, Z. Constrained percolation, Ising model and XOR Ising model on planar lattices, Random Structures and Algorithms 57 (2020), 474-525.

[9] Broadbent, S. and Hammersley, J. Percolation processes: I. Crystals and mazes, Mathematical Proceedings of the Cambridge Philosophical Society 53 (1957), 479-497. 


\section{S-/t-/p-embeddings: state-of-the-art of convergence results for the bipartite dimer and Ising models on irregular planar graphs DMitry ChelkaK}

The goal of this series of three lectures is to describe the recent progress on understanding the 'discrete conformal structure' of big planar graphs carrying either the bipartite dimer or the nearest-neighbor Ising model via their embeddings into the Minkowski spaces $\mathbb{R}^{2+2}$ or $\mathbb{R}^{2+1}$, respectively, as piece-wise linear surfaces. We briefly discuss the following topics:

1. The general framework of t-embeddings $[3,4]$, which were also introduced in [9] under the name Coulomb gauges; the special cases include:

- $s$-embeddings $[1,2]$ of graphs carrying the nearest-neighbor Ising model;

- classical barycentric or Tutte's embeddings of weighted planar graphs;

as well as even more specific ones like orthodiagonal embeddings or embeddings coming from circle patterns; e.g., see $[8,11]$.

2. The notion of t-holomorphic functions on t-embeddings $\mathcal{T}^{\delta}$ developed in [3] and key ideas of their analysis in the 'small mesh size limit' $\delta \rightarrow 0$ :

- crucial role of a space-like surface $\mathrm{S}:=(z(\zeta), \vartheta(\zeta))_{\zeta \in \mathrm{D}} \subset \mathbb{R}^{2+2}$ (where $\zeta$ stands for the conformal parametrization of $\mathrm{S}$ ), which appears as a (subsequential) limit of the graphs $\left(\mathcal{T}^{\delta} ; \mathcal{O}^{\delta}\right) \subset \mathbb{R}^{2+2}$ of origami maps $\mathcal{O}^{\delta}$;

- interpretation of the mean curvature of $\mathrm{S}$ as the mass in the propagation equation $\partial_{\bar{\zeta}} f=m \bar{f}$ for fermions appearing in the model (either bipartite dimer or nearest-neighbor Ising) under consideration;

- inevitable appearance of minimal-Lorentz surfaces $\mathrm{S}$ in the limit $\delta \rightarrow 0$, in which case the correlations of the dimer height fluctuations become harmonic in $\zeta$ and have the Gaussian structure. (If $m \neq 0$, then one should not expect that the height correlations become Gaussian in the limit $\delta \rightarrow 0$ since the bosonization of massive free fermions does not lead to free bosons; cf. [7].)

3. The notion of perfect t-embeddings or p-embeddings of finite weighted bipartite graphs; see [4]. Loosely speaking, we call a t-embedding of a finite graph (or a Coloumb gauge in the terminology of [9]) perfect if the outer face of its augmented dual belongs to the unit hyperboloid

$$
\left.\left(\mathcal{T}^{\delta} ; \mathcal{O}^{\delta}\right)\right|_{\partial_{\text {out }} G^{*}} \subset \mathrm{H}:=\left\{(z ; \vartheta):|z|^{2}=1+|\vartheta|^{2}, \vartheta \in \mathbb{R}\right\} \subset \mathbb{R}^{2+1} \subset \mathbb{R}^{2+2} .
$$

It is worth emphasizing that the existence (and uniqueness) of perfect t-embeddings remains an open question; we believe that this should be also intimately related to more 'algebraic' viewpoints on the dimer model.

Conjecture 1 ([4]). Given a (non-degenerate in a certain algebraic sense to be specified) weighted bipartite graph $G$ with the sphere topology and a marked face of a $G$, prove that its augmented dual $G^{*}$ (as defined in [9]) admits a perfect $t$ embedding and that this p-embedding is unique up to Lorentz isometries of the space $\mathbb{R}^{2+1}$ preserving the unit hyperboloid $\mathrm{H}$. 
Mini-Workshop: Dimers, Ising and Spanning Trees beyond the Critical Isoradial Case

4. The main result as well as the key ideas of our (joint with Benoît Laslier and Marianna Russkikh, in preparation) paper [4].

Let $G^{\delta}=\left(G^{\delta}, \nu^{\delta}\right), \delta \rightarrow 0$, be (a sequence of) weighted bipartite graphs with the sphere topology and with marked 'outer' faces. Assume that

(a) $\mathcal{T}^{\delta}:\left(G^{\delta}\right)^{*} \rightarrow \mathbb{C}$ are perfect $t$-embeddings of the augmented duals of $G^{\delta}$;

(b) $\mathcal{T}^{\delta}$ satisfy a 'technical' assumption ExP-FAT as $\delta \rightarrow 0$ (informally speaking this means that 'exponentially-degenerate' faces of $\mathcal{T}^{\delta}$ do not form curves of a macroscopic size; see [3] for a rigorous statement).

(c) the graphs $\left(\mathcal{T}^{\delta} ; \mathcal{O}^{\delta}\right)$ of the origami maps $\mathcal{O}^{\delta}$ (associated with $\mathcal{T}^{\delta}$ ) converge to a Lorentz-minimal surface $\mathrm{S}_{\theta} \subset \mathbb{R}^{2+1} \subset \mathbb{R}^{2+2}$ spanned by a contour

$$
\mathrm{L}_{\theta}:=\left\{\left(e^{i \phi} \cdot(\cos \theta(\phi))^{-1} ; \tan \theta(\phi)\right)\right\}_{\phi \in \mathbb{T}} \subset \mathrm{H},
$$

where $\theta: \mathbb{T} \rightarrow\left(-\frac{\pi}{2}, \frac{\pi}{2}\right)$ is a 1 -Lipschitz function on the unit circle $\mathbb{T}$.

Theorem 2 ([4]). Under the assumptions (a)-(c) listed above, the correlation functions of the fluctuations of the random dimer height functions on $\left(G^{\delta}\right)^{*}$ converge to the Gaussian Free Field (with Dirichlet boundary conditions) on the surface $\mathrm{S}_{\theta}$, where the metric on $\mathrm{S}_{\theta}$ is induced from the ambient Minkowski space.

Below are several important remarks:

(i) Recall that, as for now, the existence of p-embeddings $\mathcal{T}^{\delta}$ remains an open question. Still, it is worth mentioning that p-embeddings do exist in particular cases: e.g., in the situation when outer faces of the graphs $G^{\delta}$ have degree 4;

(ii) The existence of subsequential space-like limits $\mathrm{S} \subset \mathbb{R}^{2+2}$ of discrete surfaces $\left(\mathcal{T}^{\delta} ; \mathcal{O}^{\delta}\right)$ easily follows from compactness arguments since the definition of $\mathcal{O}^{\delta}$ implies that $\left|\mathcal{O}^{\delta}(x)-\mathcal{O}^{\delta}(y)\right| \leq\left|\mathcal{T}^{\delta}(x)-\mathcal{T}^{\delta}(y)\right|$ for all $x, y \in\left(G^{\delta}\right)^{*}$.

(iii) If we assume that such a subsequential limit $\mathrm{S}$ is a Lorentz-minimal surface, then it has to be one of the surfaces $\mathrm{S}_{\theta}$ defined above: the definition of perfect t-embeddings implies that $\mathrm{S}$ has to be bounded by a contour running in the unit hyperboloid $\mathrm{H} \subset \mathbb{R}^{2+1} \subset \mathbb{R}^{2+2}$. In particular, the minimality assumption yields that $\mathrm{S} \subset \mathbb{R}^{2+1}$, i.e., that the second coordinate of $\mathcal{O}^{\delta}$ degenerates as $\delta \rightarrow 0$.

(iv) To illustrate the general theory, in a short joint paper [5] with Sanjay Ramassamy we consider classical (homogeneous) Aztec diamonds (which are combinatorially equivalent to graphs with outer degree 4 and thus admit p-embeddings) and demonstrate that the discrete surfaces $\left(\mathcal{T}^{\delta} ; \mathcal{O}^{\delta}\right)$ converge to a surface $\mathrm{S}_{\theta}$ with a piece-wise linear function $\theta$ such that $\theta(0)=\theta(\pi)=\frac{\pi}{4}$ and $\theta\left( \pm \frac{\pi}{2}\right)=-\frac{\pi}{4}$. Of course, the metric of this surfaces gives rise to the well-known correlation functions of fluctuations in the liquid zone (and the frozen zones are collapsed to points).

(v) Recall that we do not expect that non-minimal surfaces S could lead to Gaussian height fluctuations because of the presence of the mass in the propagation equation for fermionic observables. On the other hand, the famous KenyonOkounkov prediction [10] claims that Gaussian fluctuations should appear when working with subgraphs of periodic grids, similarly to what happens for classical Aztec diamonds mentioned above. This naturally leads to the following question: 
Open question 3. What is the correspondence between the classical KenyonOkounkov theory and Lorentz-minimal surfaces? How does a concrete local structure of the graphs $G^{\delta}$ (e.g., the fact that they are pieces of the homogeneous square grid) lead to the conjectural Lorentz-minimality of limit surfaces $\mathrm{S}$ in examples similar to Aztec diamonds?

5. Finally, we briefly discuss the convergence results obtained in [2] for the nearest-neighbor Ising model on s-embeddings under the following assumptions:

- 'uniformly bounded geometry': the angles of s-embeddings $\mathcal{S}^{\delta}$ are uniformly bounded away from 0 ; the lengths of edges of $\mathcal{S}^{\delta}$ are uniformly comparable to $\delta$;

- the origami function $\mathcal{Q}^{\delta}=O(\delta)$ as $\delta \rightarrow 0$.

Certainly, these assumptions are very restrictive compared, e.g., to the very weak 'technical' assumption ExP-FAT for perfect t-embeddings mentioned above. Still, this generalizes the convergence of critical FK-Ising interfaces to SLE(16/3) curves from the very rigid classical setup of critical Baxter's Z-invariant weights on rhombic lattices [6] to the critical Ising models on arbitrary periodic graphs (via their canonical s-embeddings), circle patterns [11] etc. Let us mention that

(i) the new approach used in [2] also gives a quantitative estimate on the speed of convergence of fermionic observables; this was not known before even on $\mathbb{Z}^{2}$;

(ii) this approach avoids Smirnov's sub-/super-harmonicity trick [12] (which is not available beyond rhombic lattices) and can be adapted to the massive setup.

We also hope that the techniques developed in [2] for the analysis of discrete Riemann boundary value problems can be eventually pushed forward to drop the 'bounded geometry' assumptions but this certainly requires a non-trivial work.

\section{REFERENCES}

[1] D. Chelkak, Planar Ising model at criticality: state-of-the-art and perspectives, In Proceedings of the International Congress of Mathematicians 20183 (2018). 2789-2816.

[2] D. Chelkak. Ising model and s-embeddings of planar graphs, arXiv (2020), 2006.14559.

[3] D. Chelkak, B. Laslier, M. Russkikh, Dimer model and holomorphic functions on $t$ embeddings of planar graphs, arXiv (2020), 2001:11871.

[4] D. Chelkak, B. Laslier, M. Russkikh, Bipartite dimer model: p-embeddings and Lorentzminimal surfaces, In preparation (2021).

[5] D. Chelkak, S. Ramassamy, Fluctuations in the Aztec diamonds via a Lorentz-minimal surface, arXiv (2020), 2002.07540.

[6] D. Chelkak, S. Smirnov, Universality in the $2 D$ Ising model and conformal invariance of fermionic observables, Invent. Math. 1893 (2012), 515-580.

[7] S. Chhita, The height fluctuations of an off-critical dimer model on the square grid, J. Stat. Phys. 1482 (2012), 67-88.

[8] O. Gurel-Gurevich, D. C. Jerison, A. Nachmias, The Dirichlet problem for orthodiagonal maps, Adv. Math. 374 (2020), 53 pp.

[9] R. Kenyon, W. Y. Lam, S. Ramassamy, M. Russkikh, Dimers and circle patterns, arXiv (2018) 1810.05616.

[10] R. Kenyon, A. Okounkov, Limit shapes and the complex Burgers equation, Acta Math. 199(2) (2007), 263-302.

[11] M. Lis, Circle patterns and critical Ising models, Comm. Math. Phys. 370 (2019), 507-530. 
Mini-Workshop: Dimers, Ising and Spanning Trees beyond the Critical Isoradial

Case

[12] S. Smirnov, Conformal invariance in random cluster models. I. Holomorphic fermions in the Ising model. Ann. of Math. 1722 (2010), 1435-1467.

\section{Tau-function on Riemann surfaces.}

VLADIMIR FOCK

$\tau$-function was introduced by M.Sato [1] as a generating function for solutions of nonlinear integrable hierarchies of partial differential equations. He gave the "fermionic" interpretation for this function as a section of a determinant line bundle over infinite Grassmanian. We suggest an alternative "bosonic" definition of the $\tau$-functions corresponding to finite gap solution of integrable hierarchies.

Let $S$ be a Riemann surface. A quasi-rational function on $S$ is a function holomorphic everywhere except finitely many points and such that locally it can be written as $r e^{\phi}$ where both $r$ and $\phi$ are meropmorphic functions. The order $\operatorname{ord}_{p} r e^{\phi}$ of a quasi-rational function at a point $p \in S$ is just the order of the function $r$ at this point. Observe that quasi-rational functions are not closed with respect to addition, but nonzero quasi-rational functions form a group denoted by $\mathcal{H}(S)$ with respect to multiplication.

Local group $\mathcal{H}_{p}$. The multiplicative group of nonzero germs of quasi-rational functions in a vicinity of a point $p$ is denoted by $\mathcal{H}_{p}$. Given a local coordinate $z_{p}$ vanishing at $p$ any element of $\mathcal{H}_{p}$ can be written as

$$
f\left(z_{p}\right)=z_{p}^{n} e^{\phi\left(z_{p}\right)}=z_{p}^{n} e^{\sum t_{-i} z_{p}^{i}},
$$

where $\phi\left(z_{p}\right)$ is a germ of a meromorphic function.

The group $\mathcal{H}_{p}$ has a canonical subgroup of holomorphic germs $\mathcal{H}_{p}^{+} \subset \mathcal{H}_{p}$ taking value 1 at $p$. Elements of this group can be written as $f^{+}\left(z_{p}\right)=e^{\sum_{i>0} t_{-i} z_{p}^{i}}$. If the coordinate $z_{p}$ is fixed one can define a complimentary subgroup $\mathcal{H}_{p}^{-}$of functions of the form $f^{-}\left(z_{p}\right)=z_{p}^{n} e^{\sum_{i \leqslant 0} t_{-i} z_{p}^{i}}$. Any germ $f \in \mathcal{H}$ it can be uniquely decomposed into the product $f=f^{+} f^{-}$with $f^{+} \in \mathcal{H}_{p}^{+}$and $f^{-} \in \mathcal{H}_{p}^{-}$.

Observe that though the subgroup $\mathcal{H}_{p}^{+}$does not depend on the coordinate system, the group $\mathcal{H}_{p}^{-}$does. In other words the splitting of the exact sequence

$$
1 \rightarrow \mathcal{H}_{p}^{+} \rightarrow \mathcal{H}_{p} \rightarrow \mathcal{H}_{p} / \mathcal{H}_{p}^{+} \rightarrow 1
$$

depends on the choice of the local coordinate $z_{p}$. In particular the decomposition $f=f^{+} f^{-}$also depends on the choice of the local coordinate.

Cocycle and central extension. Define a cocycle $c_{p}: \mathcal{H}_{p} \times \mathcal{H}_{p} \rightarrow \mathbb{C}^{\times}$on the group $\mathcal{H}_{p}$ by

$$
c_{p}(f, g)=\exp \frac{1}{2 \pi i}\left(\int_{\gamma} \ln f d \ln g-\ln g(\gamma(0)) d \ln f\right),
$$

where $\gamma:[0,1] \rightarrow \mathbb{C}^{\times}$be closed path $(\gamma(0)=\gamma(1))$.

It satisfies the following properties:

- $c(f, g)$ does not depend on the choice of the path $\gamma$ and the branches of the logarithms. 
- Skew-symmetry: $c(f, g)=c(g, f)^{-1}$

- Multiplicativity: $c(f, g h)=c(f, g) c(f, h)$.

- Steinberg relation: $c(f, 1-f)=1$.

- For $f=z_{p}^{n} e^{\phi\left(z_{p}\right)}$ and $g=z_{p}^{m} e^{\psi\left(z_{p}\right)}$ it is given by

$$
c(f, g)=(-1)^{m n} \exp (\operatorname{Res} \phi d \psi+(m \phi-n \psi) d z / z)
$$

- For $f=z_{p}^{n} \exp \sum p_{-i} z_{p}^{i}$ and $g=z_{p}^{m} \exp \sum t_{-i} z_{p}^{i}$ it is given by

$$
c(f, g)=\exp \left(m p_{0}-n t_{0}+\sum i p_{i} t_{-i}\right) .
$$

- The restrictions of the cocycle to $\mathcal{H}_{p}^{+}$and to $\mathcal{H}_{p}^{-}$are equal to 1 identically.

This cocycle defines a central extension $1 \rightarrow \mathbb{C}^{\times} \stackrel{i}{\rightarrow} \hat{\mathcal{H}}_{p} \stackrel{s}{\rightarrow} \mathcal{H}_{p} \rightarrow 1$ by the multiplication rule on the set $\mathcal{H}_{p} \times \mathbb{C}^{\times}$:

$$
(f, x)(g, y)=(f g, x y c(f, g)) .
$$

Representation of $\hat{\mathcal{H}}_{p}$. The group $\hat{\mathcal{H}}_{p}$ has a subgroup $\hat{\mathcal{H}}_{p}^{+}=\left\{\left(f^{+}, x\right) \mid f^{+} \in \mathcal{H}_{p}^{+}\right\}$. It has a obvious one-dimensional representation $\hat{\mathcal{H}}_{p}^{+} \rightarrow \mathbb{C}^{\times}$with $\left(f^{+}, x\right) \mapsto x$. The representation of $\hat{\mathcal{H}}_{p}^{+}$induces a representation of the group $\hat{\mathcal{H}}_{p}$ in the space $\mathcal{B}_{p}$ of functions $v: \hat{\mathcal{H}}_{p} \rightarrow \mathbb{C}^{\times}$satisfying $v\left((g, x)\left(f^{+}, y\right)\right)=y v(g, x)$ for any $\left(f^{+}, x\right) \in \hat{\mathcal{H}}_{p}^{+}$. Such function is uniquely determined by its value on the subgroup $\mathcal{H}_{p}^{-}$. The group $\mathcal{H}_{p}$ acts on such functions by let shifts.

$$
v\left((f, x)\left(g^{-}, 1\right)\right)=x c\left(f^{+}, f^{-}\right) c^{2}\left(f^{+}, g^{-}\right) v\left(f^{-} g^{-}, 1\right) .
$$

By functions on $\mathcal{H}_{p} / \mathcal{H}_{p}^{+}$we understand functions of $k, t_{1}, t_{2}, \ldots$ with finite support with respect to $k$ and polynomial in finitely many variables out of $t_{1}, t_{2}, \ldots$.

Quasi-divisors. A quasi-divisor on $S$ is an association to every point $p \in S$ of an element of $\mathcal{H}_{p} / \mathcal{H}_{p}^{+}$in such a way that in only finitely many points these elements are nontrivial. We denote the additive $\mathbb{Z}$-graded group of quasi-divisors by $\mathrm{q} \operatorname{Div}(S)=\bigoplus \mathrm{qDiv}^{k}(S)$. The grading is given by $\operatorname{deg}_{p} r e^{\phi}=\operatorname{deg}_{p} r$.

For a quasi-rational function $f$ one can define a quasi-divisor denoted by $(f)$. The quasi-divisors obtained in this way are called principal. A principal quasidivisor on a closed surface $S$ has degree 0 . The group of principal quasi-divisors is denoted by $q \operatorname{div}(S)$. Locally any quasi-divisor is principal. Two quasi-divisors with difference belonging to $q \operatorname{div}(S)$ are called linearly equivalent. Like for ordinary divisors the space of linear equivalence classes $\mathrm{qDiv}^{k}(S) / \mathrm{qdiv}(S)=\operatorname{Pic}^{k}(S)$ is the space of line bundles over $S$ of degree $k$.

Global representation. For a surface $S$ define $\mathcal{B}(S)=\otimes_{p \in S} \mathcal{B}_{p}$. If we fix local coordinates around all points, the elements of $\mathcal{B}(S)$ can be considered as functions on quasi-divisors $\mathrm{q} \operatorname{Div}(S)$. Observe that the non-extended group $\mathcal{H}(S)$ is a subgroup of $\widehat{\otimes_{p} \mathcal{H}_{p}}$ and therefore it acts on $\mathcal{B}(S)$. Indeed, it is a consequence of the Weil reciprocity formula extended to quasi-meromorphic functions:

$$
\prod_{p \in S} c_{p}(f, g)=1
$$


Mini-Workshop: Dimers, Ising and Spanning Trees beyond the Critical Isoradial Case

Definition of the $\tau$-function : $\tau$-function is just the invariant of the group the space $\left.\mathbb{B}_{(} S\right)$ under the action of $\mathcal{H}(S)$. In the representation of $\mathcal{B}(S)$ as functions on $\mathrm{qDiv}^{0}$ the invariance of $\tau$ reads as

$$
\tau[d]=\tau[d+(f)] \prod_{p \in S} c_{p}^{2}\left(f^{+}, g_{p}(d)\right) c_{p}\left(f^{+}, f^{-}\right),
$$

where $g_{p}(d)$ is a representative of $d$ in the vicinity of $p$.

\section{Properties of the $\tau$-function :}

A. The equation (1) defines the $\tau$-function uniquely up to a multiplicative constant.

B. The $\tau$ function satisfies Hirota bilinear relations which we do not formulate here in full generality.

C. The value of the $\tau$-function on a divisor supported at one point coincides with the standard $\mathrm{KP} \tau$-function. Its value on a divisor on supported on two points with $k=1, t_{i}=0$ in one of the points is the KP Baker-Akhiezer function [2]. Similarly $\tau$ - and Baker-Akhiezer functions for SG and KdV hierarchies are particular values of our $\tau$-function.

D. The values of the $\tau$-function on ordinary divisors is given by an explicit formula in terms of Riemann theta function:

$$
\tau\left[\sum n_{i}\left[z_{i}\right]\right]=\theta_{q}\left(t+\sum n_{i} z_{i}\right) \prod_{i<j} \theta_{q}\left(z_{i}-z_{j}\right)^{n_{i} n_{j}}
$$

These values satisfy discrete Hirota bilinear equation

$$
\begin{array}{r}
\tau\left[d+z_{1}+z_{2}\right] \tau\left[d+z_{3}+z_{4}\right]+\tau\left[d+z_{1}+z_{3}\right] \tau\left[d+z_{2}+z_{4}\right]+ \\
+\tau\left[d+z_{1}+z_{4}\right] \tau\left[d+z_{2}+z_{3}\right]=0,
\end{array}
$$

where $d$ is a divisor of degree -2 . They and explicit expression $\mathcal{A}$-coordinates for cluster integrable systems.

E. The function $\tau[d]$ can be viewed as a limit of the generating function $S$ : $\Omega^{01}(S) \rightarrow \mathbb{C}$ of the Lagrangian variety of closed 1-forms in the symplectic variety of all forms. The function $S$ satisfies the equation

$$
S(\alpha)=S(\alpha+\partial \ln f) \exp \int \bar{\partial} \ln f \partial \ln f+2 \alpha \partial \ln f
$$

for any smooth function $f$. The limit of this relation when the form $\alpha$ is a distribution valued form with support at finitely many points gives the relation (1).

\section{REFERENCES}

[1] M. Sato, Soliton equations as dynamical systems on infinite-dimensional Grassmann manifold, RIMS Kokyuroku (1981) 439, 30-46.

[2] B.A. Dubrovin, Theta functions and non-linear equations, Russ. Math. Surv. (1981) 362. 


\section{Elliptic dimer models and genus 1 Harnack curves David Cimasoni \\ (joint work with Cédric Boutillier and Béatrice de Tilière)}

The aim of this talk was to present an ongoing research project with Cédric Boutillier and Béatrice de Tilière $[1,2,3]$.

Almost 20 years ago, Kenyon [6] showed that dimers on a bipartite isoradial graph G with critical weights admit a Gibbs measure that is local, in the sense that the probability of any finite family of edges of $G$ being occupied by dimers only depends on the geometry of $G$ near these edges. However, such weighted graphs form a very specific class: in the periodic case, they correspond to rational Harnack curves, i.e. Harnack curves of genus 0 , and the aforementioned measure is one among the two-parameter family of ergodic Gibbs measures that can be defined for such a curve [8]. Therefore, this phenomenon has long been considered as rather exceptional.

Our project can be understood as a wide-reaching extension of Kenyon's remarkable results, in two directions. First, we do not restrict ourselves to graphs that can be isoradially embedded, which by [9] are exactly those planar graphs whose train-tracks form neither self-intersections nor bigons. Instead, we consider the wider class of minimal graphs [10] given by planar bipartite graphs whose (consistently oriented) train-tracks do not self-intersect and do not form parallel bigons. As proved in [1], a graph is minimal if and only if it admits a minimal immersion, a concept generalising that of isoradial embedding. Moreover, the space of such immersions can be described as an explicit subset of the space of angles maps associated to oriented train-tracks. One of the main results of [1] is that minimal graphs with such angle maps give the correct framework to study dimer models with the aim of locality in mind. Secondly, we do not restrict ourselves to the genus 0 case, but consider Fock's weights [4] in the elliptic (i.e. genus 1) case in [2], and plan to address the case of arbitrary genus soon [3].

The introduction of Fock's weights deserves some context. It was known since Kenyon-Okounkov [7] and Goncharov-Kenyon [5] that dimer models on minimal graphs allow to realise any spectral data, i.e. any Harnack curve together with a divisor given by one point on each oval. The construction of a minimal graph with the correct Newton polygon is not such a difficult problem, but it was not clear how to construct the correct dimer weights on such a graph. In [4], Fock starts with an arbitrary smooth planar curve of degree $g$ (not necessarily a Harnack curve) together with a degree $g$ divisor on it (not necessarily on its ovals), and explicitly constructs weights on the minimal graph so that the corresponding "dimer model" has this "spectral data".

The first contribution of [2] is to show how to tune the parameters in Fock's elliptic weights in order for the corresponding operator $\mathrm{K}$ to be Kasteleyn: one needs to start with a rectangular torus (i.e. to fix $\tau$ purely imaginary, so that the real locus consists of two components), to chose the divisor to be a point $t$ 
Mini-Workshop: Dimers, Ising and Spanning Trees beyond the Critical Isoradial

Case

on one of these components, and to fix an angle map $\alpha$ defining a minimal immersion of the minimal graph. With these hypotheses, we then obtain a family of inverses $\left\{A^{u_{0}}\right\}_{u_{o} \in D}$ for the Kasteleyn operator $\mathrm{K}$, indexed by the upper half $D$ of the torus with modular parameter $\tau$. The most remarkable property of these inverses is that they are local, in the sense that $A_{b, w}^{u_{0}}$ only depends on the geometry of the graph near a path joining $b$ and $w$.

For our next result, we need a technical assumption that holds trivially for periodic graphs, and that we expect to hold for any minimal graph. If this condition is satisfied, then for every $u_{0} \in D$, the operator $\mathrm{A}^{u_{0}}$ defines a Gibbs measure on dimer configurations of $\mathrm{G}$. Furthermore, the set $D$ gives the phase diagram of the model: when $u_{0}$ is on the top boundary of $D$, the dimer model is gaseous (exponential decay of correlations); when $u_{0}$ is in the interior of the set $D$, the model is liquid (polynomial decay of correlations); when $u_{0}$ is on the lower boundary of $D$, the model is solid (no decay of correlations). Note that when G is periodic, this gives an alternative description of the full set of ergodic Gibbs measures of [8]. Remarkably, these measures are now given by explicit local formulas. By aforementioned work of Goncharov-Kenyon, it is no longer difficult to show that every genus 1 Harnack curve with a marked point on the oval is the spectral data of an explicit dimer model on a minimal graph $\mathrm{G}$ with Fock's elliptic Kasteleyn operator, for a unique parameter $t$, and an angle map $\alpha$ defining a minimal immersion of $\mathrm{G}$.

Extending these arguments to the case of arbitrary genus, we now believe that the possibility of expressing locally an ergodic Gibbs measure on dimers is not the exception, but the rule.

\section{REFERENCES}

[1] Cédric Boutillier, David Cimasoni, and Béatrice de Tilière, Isoradial immersions, arXiv (2019), 1912.10297.

[2] Cédric Boutillier, David Cimasoni, and Béatrice de Tilière, Elliptic dimers on minimal graphs and genus 1 Harnack curves, arXiv (2020), 2007.14699.

[3] Cédric Boutillier, David Cimasoni, and Béatrice de Tilière, Dimers on minimal graphs and genus g Harnack curves, (2020+)

[4] Vladimir V. Fock, Inverse spectral problem for GK integrable system, arXiv (2015), 1503.00289.

[5] Alexander B. Goncharov and Richard Kenyon, Dimers and cluster integrable systems, Ann. Sci. Éc. Norm. Supér. (2013) 46(5):747-813.

[6] Richard Kenyon, The Laplacian and Dirac operators on critical planar graphs, Invent. Math. (2002) 150(2):409-439.

[7] Richard Kenyon and Andrei Okounkov, Planar dimers and Harnack curves, Duke Math. J. (2006) 131(3):499-524.

[8] Richard Kenyon, Andrei Okounkov, and Scott Sheffield, Dimers and amoebae, Ann. of Math. (2) (2006) 163(3):1019-1056.

[9] Richard Kenyon and Jean-Marc Schlenker, Rhombic embeddings of planar quad-graphs, Trans. Amer. Math. Soc. (2005) 357(9):3443-3458 (electronic).

[10] Dylan P. Thurston, From dominoes to hexagons, In Proceedings of the 2014 Maui and 2015 Qinhuangdao conferences in honour of Vaughan F. R. Jones' 60th birthday (2017) 46 of Proc. Centre Math. Appl. Austral. Nat. Univ., 399-414. 


\section{The multidimer model \\ RICHARD KENYON \\ (joint work with Andrei Pohoata)}

The dimer model on a graph is the probability model of random perfect matchings of that graph. For arbitrary graphs essentially nothing is known about it, since even computing the number of configurations is NP hard (it is equivalent to computing the permanent).

We present a certain limit of the dimer model in which exact calculations can be done.

The multidimer model on a graph $\mathcal{G}$ is the dimer model on a "blow up" $\mathcal{G}_{\mathbf{N}}$ of $\mathcal{G}$, where $\mathcal{G}_{\mathbf{N}}$ is obtained from $\mathcal{G}$ by replacing each vertex $v$ with $N_{v}$ vertices and each edge $u v$ with a complete bipartite graph $K_{N_{u}, N_{v}}$. Here $\mathbf{N}=\left\{N_{v}\right\}_{v \in V}$ is an assignment of multiplicities to vertices of $\mathcal{G}$.

If we assign positive weights $c_{e}$ to edges of $\mathcal{G}$, then there is a natural probability measure on dimer covers of $\mathcal{G}_{\mathbf{N}}$, giving a dimer cover $m$ a probability proportional to the product of its edge weights.

The partition function $Z(\mathbf{N})$ of the multidimer model has a surprisingly simple exponential generating function, which is just $\exp (P)$ where $P=\sum_{u \sim v} c_{u v} x_{u} x_{v}$ is the "edge polynomial".

In the limit of large multiplicities we can compute the free energy of the multidimer model on arbitrary graphs. This follows from a saddle-point calculation. We also show that the edge occupation process tends to the Gaussian free field with respect to an associated graph Laplacian.

Furthermore we also solve an analog of the monomer/dimer problem in this setting, by changing the multiplicities slightly away from constants. In this case the excess or deficit multiplicity acts like a charge associated to a particle at that vertex, and these charges repel or attract via a Coulomb potential on the graph.

One of the most interesting features of the usual single dimer model is its limit shape theory. For regular geometric graphs like $\mathbb{Z}^{d}$ we can give an analogous limit shape theory, along with an explicit surface tension function.

Triple crossing diagrams, projective configurations, dimers Niklas AfFolter

(joint work with Max Glick, Pavlo Pylyavskyy and Sanjay Ramassamy)

The goal is to study a wide array of maps from discrete differential geometry, discrete integrable systems and statistical mechanics with one framework, that captures and relates the geometric and algebraic properties of these maps. To this end we introduce TCD maps, which associate certain configurations in $\mathbb{C P}^{n}$ to triple crossing diagrams [1]. To every such map we can associate a dimer partition function [2], with face weights that are invariant under projective transformations. Note that in general, the edge and face weights of these partition functions may not necessarily be real positive. Moreover, we can locally change the combinatorics of 
Mini-Workshop: Dimers, Ising and Spanning Trees beyond the Critical Isoradial

Case

TCD maps. Such a change of combinatorics is closely related to Menelaus' theorem in geometry, while it corresponds to a spider move in statistical physics. The setup of TCD maps allows us to find dimers associated to the pentagram map (as found previously by Glick [3]), to objects from discrete differential geometry and discrete integrable systems like Q-nets, Darboux maps and Line complexes, A-nets, Cox lattices and so forth. In fact, we can also algebraically extend these definitions to $\mathbb{C P}^{1}$, where the maps are not well defined by their geometry anymore. In a bit of a surprise, it turns out that t-embeddings, harmonic embeddings and s-embeddings known from statistical physics are not only special cases of TCD maps, but of Qnets, Darboux maps and Line complexes. However, the dimer partition functions that we have associated to TCD maps do not correspond to the previously known $[4,5]$ dimer partition functions associated to t-embeddings, harmonic embeddings and s-embeddings. This has motivated us to find a second dimer partition function associated to a TCD map. This partition function is not invariant under general projective transformations, but only under affine transformations. It reproduces the partition functions occurring in t-embeddings, harmonic embeddings and sembeddings and also in T-graphs. In fact, by carefully studying the effects of projections, sections and duals we can associate $(n+2)$ dimer partition functions to any TCD map in $\mathbb{C P}^{n}$. While many cases of TCD maps with real positive face weights are known, no general, systematic investigation exists. Therefore, conjecturally, a wide array of TCD maps exist (in $\mathbb{C P}^{1}$ and beyond) that feature the dimer model, but have not been investigated at all.

Moreover, it is well known that for certain combinatorics, one can study spanning trees and the Ising model via the resistor subvariety and the Ising subvariety, where we consider subvarieties of the face weights of the dimer model. It turns out that these subvarieties can be translated to conditions on the geometry of TCD maps. In the projective case these are the so called Konigs nets and CKP Darboux maps. In fact we can show that many TCD maps that are known to feature a discrete BKP equation have dimer face weights in the resistor subvariety, while TCD maps that are known to feature a discrete CKP equation have dimer face weights in the Ising subvariety. Additionally, we also show that if we begin with TCD maps that satisfy additional quadratic constraints with respect to a symmetric (or anti-symmetric) bilinear form in $\mathbb{C P}^{3}$, we obtain TCD maps with variables in the resistor (or Ising) subvariety. As special cases we recover harmonic embeddings and s-embeddings. An open question is, whether there is a conceptual reason why symmetric bilinear forms lead to the resistor subvariety and the BKP equation while anti-symmetric bilinear forms lead to the Ising subvariety and the CKP equation. Moreover, it is unclear if there are other subvarieties occurring for TCD maps in particular position to bilinear forms in higher dimensional spaces $\left(\right.$ than $\left.\mathbb{C P}^{3}\right)$.

\section{REFERENCES}

[1] Thurston, Dylan P. From Dominoes to Hexagons, Proceedings of the 2014 Maui and 2015 Qinhuangdao Conferences in Honour of Vaughan F.R. Jones 60th Birthday (2017), 399-414. 
[2] Niklas Affolter and Max Glick and Pavlo Pylyavskyy and Sanjay Ramassamy Vector-relation configurations and plabic graphs, arXiv (2019), 1908.06959.

[3] Max Glick The pentagram map and Y-patterns, Advances in Mathematics 227 (2011), 1019-1045.

[4] R. Kenyon, W. Y. Lam, S. Ramassamy and M. Russkikh, Dimers and circle patterns, arXiv (2018), 1810.05616.

[5] Niklas C. Affolter, Miquel Dynamics, Clifford Lattices and the Dimer Model, arXiv (2018), 1808.04227.

\section{The Dirichlet problem for orthodiagonal maps}

ASAF NACHMIAS

(joint work with Ori Gurel-Gurevich and Daniel C. Jerison)

In [6] we prove that the discrete harmonic function corresponding to smooth Dirichlet boundary conditions on orthodiagonal maps, that is, plane graphs having quadrilateral faces with orthogonal diagonals, converges to its continuous counterpart as the mesh size goes to 0 . This provides a convergence statement for discrete holomorphic functions, similar to the one obtained by Chelkak and Smirnov [1] for isoradial graphs. By the double circle packing theorem, any finite, simple, 3-connected planar map admits an orthodiagonal representation.

Our result improves the work of Skopenkov [11] and Werness [13] by dropping all regularity assumptions required in their work and providing effective bounds. In particular, no bound on the vertex degrees is required. Thus, the result can be applied to models of random planar maps that with high probability admit orthodiagonal representation with mesh size tending to 0 . In a companion paper [5], we show that this can be done for the discrete mating-of-trees random map model of Duplantier, Gwynne, Miller and Sheffield [3, 7].

Discrete complex analysis is a powerful tool in the study of two-dimensional statistical physics. It has been employed to prove the conformal invariance of the scaling limit of critical percolation and the critical Ising/FK model, see Smirnov's ICM survey [12]. The high-level program of such proofs is to 1) find a modeldependent function (the so-called discrete parafermionic observable) on the lattice which satisfies some discrete version of the Cauchy-Riemann equations; 2) use discrete complex analysis to show that as the lattice's mesh size tends to 0 , the discrete observable converges to a continuous holomorphic function; 3 ) identify this function uniquely by its boundary values. The results obtained this way include some of the most remarkable breakthroughs in contemporary probability theory.

Here we address the second part of the program above, namely, the convergence of discrete harmonic or holomorphic functions to their continuous counterparts. This study has been performed on the square lattice [2] as well as on rhombic lattices [1], which are plane graphs such that each inner face is a rhombus. Because not every quadrangulation can be embedded in $\mathbb{C}$ as a rhombic lattice [8], Smirnov asked, "can we always find another embedding with a sufficiently nice version of discrete complex analysis?" [12, Section 6, Question 1]. 
Mini-Workshop: Dimers, Ising and Spanning Trees beyond the Critical Isoradial

Case

A broader class than the rhombic lattices are the orthodiagonal maps: plane graphs whose inner faces are quadrilaterals with orthogonal diagonals. See Figure 1. One can ask which planar maps are representable by a rhombic lattice or by an orthodiagonal map. For rhombic lattices the answer is the isoradial graphs. Unfortunately, these do not include all finite simple triangulations [8]. By contrast, every finite simple triangulation has an orthodiagonal representation which can be constructed using circle packing. We observe that the double circle packing theorem provides an orthodiagonal representation for any simple, 3-connected finite planar map.

Skopenkov [11] proved a convergence result for the Dirichlet problem on orthodiagonal maps under certain local and global regularity conditions. Werness [13] improved this result to assume only local regularity. All these works require a uniform bound on the vertex degrees of the maps.

The main result, Theorem 1, is a convergence statement that has no regularity assumptions of any kind. In particular, our result applies even when the vertex degrees are not uniformly bounded. Our only condition is that the maximal edge length of the map tends to 0 . As well, our proof avoids compactness arguments and thus provides an effective bound for the convergence.

Removing the regularity assumptions is not just mathematically pleasing; rather, it provides a framework for the study of discrete complex analysis on random planar maps $[9,10]$. In order to apply Theorem 1 to a given random map model, one has to verify the maximal edge length condition above. This condition is believed to hold in all natural random map models, though proving it for a random simple triangulation on $n$ vertices is considered an important open problem (see [9, Section 6]). In the companion paper [5] we show that it indeed holds for the discrete mating-of-trees random map model of Duplantier-Gwynne-Miller-Sheffield [3, 7]. Hence Theorem 1 can be applied to this model; see [5].

There has been a great deal of interest in recent years in studying statistical physics models, such as percolation, Ising/FK and the self-avoiding walk, on random planar maps. The behavior of these models at their critical temperature is mysteriously related via the KPZ correspondence to their behavior on the usual square or triangular lattices [4]. A very ambitious program is to rigorously relate the behavior of a statistical physics model in the random planar map setting (where in many cases the model is tractable) to its behavior on a regular lattice. We hope that the framework for discrete complex analysis on random planar maps that we provide in this paper will be useful for this endeavor.

Below is the statement of our main theorem. Even though some of the notation has not been defined, the conclusion should be clear: the discrete harmonic function is close to the continuous one when the mesh size is small. We have gathered the necessary definitions required to parse this theorem in Section 1.1 of [6].

Theorem 1 ([6]). Let $\Omega \subset \mathbb{R}^{2}$ be a bounded simply connected domain, and let $g: \mathbb{R}^{2} \rightarrow \mathbb{R}$ be a $C^{2}$ function. Given $\varepsilon, \delta \in(0, \operatorname{diam}(\Omega))$, let $G=\left(V^{\bullet} \sqcup V^{\circ}, E\right)$ be a finite orthodiagonal map with maximal edge length at most $\varepsilon$ such that the Hausdorff distance between $\partial G$ and $\partial \Omega$ is at most $\delta$. Let $h_{c}: \bar{\Omega} \rightarrow \mathbb{R}$ be the 


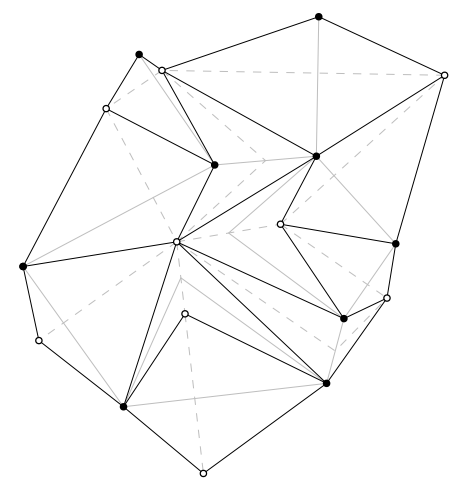

Figure 1. An orthodiagonal map. Primal vertices are represented by solid disks $\bullet$ and dual vertices by hollow disks $\circ$. The edges of the orthodiagonal map itself are drawn in black. Edges of the primal graph are drawn with solid gray lines, and edges of the dual graph are drawn with dashed gray lines.

solution to the continuous Dirichlet problem on $\Omega$ with boundary data $g$, and let $h_{d}: V^{\bullet} \rightarrow \mathbb{R}$ be the solution to the discrete Dirichlet problem on $\operatorname{Int}\left(V^{\bullet}\right)$ with boundary data $\left.g\right|_{\partial V^{\bullet}}$. Set

$$
C_{1}=\sup _{x \in \widetilde{\Omega}}|\nabla g(x)|, \quad C_{2}=\sup _{x \in \widetilde{\Omega}}\|H g(x)\|_{2}
$$

where $\widetilde{\Omega}=\operatorname{conv}(\bar{\Omega} \cup \widehat{G})$. Then there is a universal constant $C<\infty$ such that for all $x \in V^{\bullet} \cap \bar{\Omega}$

$$
\left|h_{d}(x)-h_{c}(x)\right| \leq \frac{C \operatorname{diam}(\Omega)\left(C_{1}+C_{2} \varepsilon\right)}{\log ^{1 / 2}(\operatorname{diam}(\Omega) /(\delta \vee \varepsilon))}
$$

\section{REFERENCES}

[1] D. Chelkak and S. Smirnov, Discrete complex analysis on isoradial graphs, Adv. Math. 228 (2011), 1590-1630.

[2] R. Courant, K. Friedrichs, and H. Lewy, Über die partiellen Differenzengleichungen der mathematischen Physik, Math. Ann. 100 (1928), 32-74.

[3] B. Duplantier, J. Miller, and S. Sheffield, Liouville quantum gravity as a mating of trees, arXiv (2014), 1409.7055

[4] B. Duplantier and S. Sheffield, Liouville quantum gravity and KPZ, Invent. Math. (2011) 185, 333-393.

[5] O. Gurel-Gurevich, D. C. Jerison, and A. Nachmias, A combinatorial criterion for macroscopic circles in planar triangulations, arXiv (2019), 1906.01612.

[6] O. Gurel-Gurevich, D. C. Jerison, and A. Nachmias, The Dirichlet problem for orthodiagonal maps, Adv. Math. (2020) 374, 53.

[7] E. Gwynne, J. Miller, and S. Sheffield, The Tutte embedding of the mated-CRT map converges to Liouville quantum gravity, arXiv (2017), 1705.11161.

[8] R. Kenyon and J.-M. Schlenker, Rhombic embeddings of planar quad-graphs, Trans. Amer. Math. Soc. (2005) 357, 3443-3458. 
Mini-Workshop: Dimers, Ising and Spanning Trees beyond the Critical Isoradial Case

[9] J.-F. Le Gall, Random geometry on the sphere, In Proceedings of the International Congress of Mathematicians-Seoul 2014. Vol. 1 (2014) 421-442.

[10] G. Miermont, Aspects of random maps, Saint-Flour lecture notes (2014) http://perso.enslyon.fr/gregory.miermont/coursSaint-Flour.pdf.

[11] M. Skopenkov, The boundary value problem for discrete analytic functions Adv. Math. (2013) 240 61-87.

[12] S. Smirnov, Discrete complex analysis and probability, In Proceedings of the International Congress of Mathematicians. Volume I (2010) 595-621.

[13] B. M. Werness, Discrete analytic functions on non-uniform lattices without global geometric control, arXiv (2015), 1511.01209.

\section{Cube flips in s-embeddings and $\alpha$-immersions \\ Paul Melotti}

(joint work with Sanjay Ramassamy and Paul Thévenin)

Our aim is to discuss a property that should be satisfied by "canonical" embeddings of planar graphs equipped with a certain model. This property, which we call here a cube flip, states that if the model undergoes a star-triangle transformation (or Y- $\Delta$ move) that leaves the measure unchanged, then the corresponding embeddings of the star-graph $G_{\Delta}$ and the triangle-graph $G_{Y}$ should differ only locally. In other words, a coupling property is conjugated with a theorem of planar geometry.

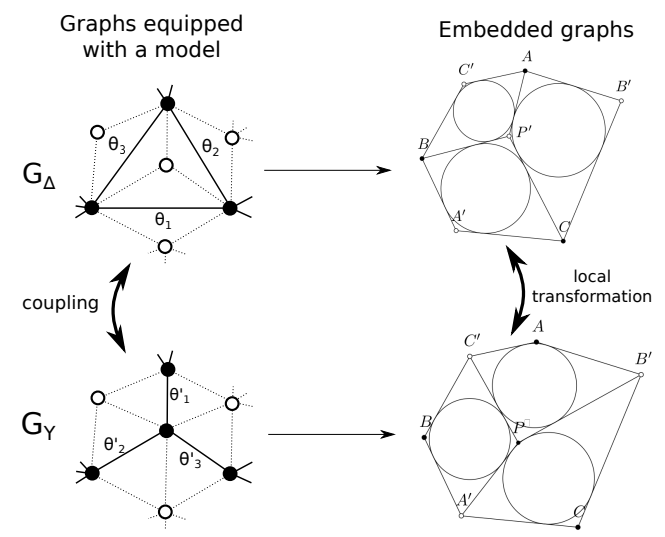

FigurE 1. The cube flip property (for s-embeddings)

This is in contrast with the usual requirements for embeddings of graphs equipped with model, which is that observables should converge to universal conformally invariant objects. Here we are interested only in a local feature. It is to be noted that all embeddings known at the moment seem to display both these global and local properties. 
The first case we discuss is the notion of harmonic embeddings, first introduced by Tutte, that maps a graph equipped with positive conductances into an arrangement of orthodiagonal quadrilaterals. Those are the quadrilaterals with successive side lengths $a, b, c, d$ such that $a^{2}+c^{2}=b^{2}+d^{2}$. In this setting, the usual startriangle transformation of resistor networks is conjugated with a theorem of planar geometry apparently due to Steiner; this was first noted by Konopelchenko and Schief [3].

Theorem 1 (Steiner, [3]). Let $G_{\Delta}, G_{Y}$ be graphs equipped with conductances related by a star-triangle move. For any Tutte embedding of $G_{\Delta}^{\diamond}$, there exists a unique Tutte embedding of $G_{Y}^{\diamond}$ that differs only at the central point.

It was also proved by Kenyon, Lam, Ramassamy and Russkikh [2] that this transformation can be seen at the level of the t-embedding of the underlying dimer model given by Temperley's bijection, and can thus be written as a composition of Miquel moves.

We then turn our attention to s-embeddings, which correspond to the Ising model, were introduced by Chelkak [1] and are the object of active research. An s-embedding maps the graph to an arrangement of tangential quadrilaterals, which is equivalent to them satisfying $a+c=b+d$. We say that an embedding is proper if it respects a fixed order around every face of the initial graph.

Theorem 2 ([4]). Let $G_{\Delta}, G_{Y}$ be graphs equipped with Ising models related by a star-triangle move. For any proper s-embedding of $G_{\Delta}^{\diamond}$, there exists a unique proper s-embedding of $G_{Y}^{\diamond}$ that differs only at the central point.

The existence can be proved using the machinery of s-embeddings. However, this does not yield uniqueness. We provide a fully geometric proof of the latter. One could also get uniqueness by using a composition of Miquel moves.

As a consequence the proof of Theorem 2, we provide a new concise formula for the star-triangle move of the Ising model:

Theorem $3([4])$. Let $\left(G_{\Delta}, \theta\right),\left(G_{Y}, \theta^{\prime}\right)$ be graphs equipped with Ising models and related by a star-triangle move. The Ising models on $G_{\Delta}$ and $G_{Y}$ are equal in distribution if

$$
\begin{gathered}
\cos \theta_{1}^{\prime}=\frac{\sin \theta_{1} \cos \theta_{2} \cos \theta_{3}}{\sin \theta_{1}+\sin \theta_{2} \sin \theta_{3}}, \cos \theta_{2}^{\prime}=\ldots, \cos \theta_{3}^{\prime}=\ldots \\
\left(\text { or equivalently, } \sin \theta_{1}=\frac{\cos \theta_{1}^{\prime} \sin \theta_{2}^{\prime} \sin \theta_{3}^{\prime}}{\cos \theta_{1}^{\prime}+\cos \theta_{2}^{\prime} \cos \theta_{3}^{\prime}}, \ldots\right)
\end{gathered}
$$

In a third part of the talk, we introduce the notion of $\alpha$-quads as those that satisfy $a^{\alpha}+c^{\alpha}=b^{\alpha}+d^{\alpha}$, for some $\alpha \in \mathbb{R}^{*}$; this can be extended naturally to $\alpha=0$ with $a c=b d$, to $\alpha=+\infty$ with $\max (a, c)=\max (b, d)$, and to $\alpha=-\infty$ with $\min (a, c)=\min (b, d)$. After giving a brief overview of the space of $\alpha$-quads when $\alpha$ runs through $\mathbb{R} \cup\{ \pm \infty\}$, we introduce $\alpha$-immersions as graph immersions into $\alpha$-quads. These being immersions and not embeddings, edges may intersect. 
Mini-Workshop: Dimers, Ising and Spanning Trees beyond the Critical Isoradial

Case

Theorem 4 ([4]). Let $\alpha>1$. For any $\alpha$-immersion of $G_{\Delta}^{\diamond}$, there exists an $\alpha$ immersion of $G_{Y}^{\diamond}$ that differs only at the central point.

This theorem is expected to be false for $\alpha \leq 1$, and we provide counter-examples for $\alpha$ close to 0 . Moreover, we do not claim uniqueness of the central point, and we can produce counter-examples with as many as 3 potential central points. We conjecture this to be the maximal number.

The proof of Theorem 4 relies on the introduction $\alpha$-curves, which are sets

$$
\left\{M \mid M A^{\alpha}-M B^{\alpha}=\lambda\right\}
$$

for two fixed points $A, B$ and $\lambda \in \mathbb{R}$. Analysing the asymptotic behaviour of these curves yields the statement.

This leaves a few questions open:

- Do $\alpha$-immersions correspond to some model of statistical mechanics, or to some integrable system, for some $\alpha$ distinct from 1 and 2 ?

- Do other families of quads exhibit geometric properties analogous to cube moves? Namely, one may look at $f$-quads, defined for a homogeneous symmetric function $f$ by $f(a, c)=f(b, d)$.

- The case $\alpha=0$ may be worth special investigation. In this case, $\alpha$-curves are simply circles, and an analogous to a cube flip might be obtained by replacing points with radical axis of circles.

\section{REFERENCES}

[1] D. Chelkak, Planar Ising model at criticality: state-of-the-art and perspectives, Proc. Int. Cong. of Math. - Rio de Janeiro (2018) IV 2801-2828.

[2] R. Kenyon, W. Y. Lam, S. Ramassamy and M. Russkikh, Dimers and circle patterns, arXiv (2019), 1810.05616.

[3] B. G. Konopelchenko and W. K. Schief, Reciprocal figures, graphical statics, and inversive geometry of the Schwarzian BKP hierarchy, Stud. Appl. Math. (2002) 109 89-124.

[4] P. Melotti, S. Ramassamy and P. Thévenin, Cube moves for s-embeddings and $\alpha$-realizations, arXiv (2020), 2003.08941.

\section{Inverse spectral problem for biperiodic networks TERrence George}

A planar electrical network is a pair $(G, c)$ where $G$ is a planar graph and $c$ is a function that associates a non-zero complex number to each edge of $G$, defined modulo overall scaling by a constant, called its conductance. Planar electrical networks provide the setting for discrete potential theory and are intimately related to random walks, spanning trees and discrete geometry. We say that $(G, c)$ is biperiodic, if it is invariant under a translation action of $\mathbb{Z}^{2}$. In this case, we take the quotient $(G, c) / \mathbb{Z}^{2}$ to get an electrical network on a torus.

We start by defining the space of electrical networks. To each graph $G$ on the torus that satisfies a certain topological condition called minimality, we associate 
the space $\mathcal{R}_{G}=\left(\mathbb{C}^{*}\right)^{\text {number of edges of } G-1}$ of conductances on it. There is a local rearrangement of networks called the $Y-\Delta$ transformation that preserves all electrical properties outside the region where the transformation happens. For each convex integral polygon $N$ in the plane that centrally symmetric, that is, invariant under rotation by $\pi$, a construction of Goncharov and Kenyon [6] gives a family of electrical networks associated to $N$ that are related by $Y-\Delta$ moves. A sequence of $Y-\Delta$ moves relating any two networks $(G, c)$ and $\left(G^{\prime}, c^{\prime}\right)$ in this family induces a birational map between $\mathcal{R}_{G}$ and $\mathcal{R}_{G^{\prime}}$ mapping $c$ to $c^{\prime}$. Gluing the spaces $\mathcal{R}_{G}$ for all networks $G$ in the family according to these birational maps, we obtain the resistor network cluster variety $\mathcal{R}_{N}$ parameterizing electrical networks associated to the polygon $N$.

The fundamental operator in the study of electrical networks is the discrete Laplacian. Associated to the discrete Laplacian on a biperiodic network is its spectral transform which is a rational map from $\mathcal{R}_{N}$ to a certain moduli space of curves and divisors. We show that this moduli space is a family of Prym varieties and show that the spectral transform is birational. This provides a classfication of biperiodic networks, analogous to the classification of electrical networks in a disc in terms of response matrices by Curtis, Ingerman and Morrow [3].

Spectral curves of genus zero correspond to isoradial networks. In this case, our inverse spectral transform recovers Kenyon's expressions in [7] for the isoradial conductances in terms of tangents. Boutillier, de Tilire and Raschel [2] have extended isoradial networks in a different way to the setting of massive isoradial networks. It would be very interesting to extend the spectral transform to the massive non-isoradial case, generalizing both.

A sequence of $Y-\Delta$ moves that takes a graph $G$ to itself gives rise to a birational automorphism of $\mathcal{R}_{N}$ called a cluster modular transformation. We show that cluster modular transformations are translations in the Prym variety, which hints that $\mathcal{R}_{N}$ is an integrable system. Our results for electrical networks parallel Fock's results in [4] for the dimer model, where the associated cluster variety is the phase space of the dimer integrable system of Goncharov and Kenyon [6]. A natural question here is whether electrical networks are Liouville integrable. We do not have a Poisson structure on $\mathcal{R}_{N}$ but a possible approach to finding it is as follows: the Poisson structure of the dimer integrable system is known to be induced by the Poisson-Lie structure of GL from work of Fock and Marshakov [5]. For electrical networks in a disc, Lam and Pylyavskyy [8] showed that there is a natural action of a symplectic group that is analogous to the action of GL on dimers. We expect that the Poisson structure on $\mathcal{R}_{N}$ that we want is induced by the Poisson-Lie structure of the symplectic group.

\section{REFERENCES}

[1] Terrence George Spectra of biperiodic planar networks, arXiv (2019), 1901.06353. 
Mini-Workshop: Dimers, Ising and Spanning Trees beyond the Critical Isoradial Case

[2] Boutillier C., de Tilière B., Raschel K. The Z-invariant massive Laplacian on isoradial graphs. Invent. math. 208 (2017), 109.

[3] Curtis E. B., Ingerman D., Morrow J. A. Circular planar graphs and resistor networks. Linear Algebra Appl. 283 (1998), 115-150.

[4] Fock V. V.: Inverse spectral problem for GK integrable systems. arXiv (2015), 1503.00289.

[5] Fock V., Marshakov A. Loop Groups, Clusters, Dimers and Integrable Systems. Geometry and Quantization of Moduli Spaces. Advanced Courses in Mathematics. Birkhäuser, Cham (2016).

[6] Alexander B. Goncharov and Richard Kenyon, Dimers and cluster integrable systems, Ann. Sci. Éc. Norm. Supér. 46 (2013), 747-813.

[7] Kenyon R. The Laplacian and Dirac operators on critical planar graphs. Invent. Math. 150 (2002), 409-439.

[8] Lam T., Pylyavskyy P. Laurent phenomenon algebras. Cambridge Journal of Mathematics 4 (2016), 121-162. 